\title{
Search for flavor-changing non-standard neutrino interactions by MINOS
}

P. Adamson, ${ }^{8}$ G. Barr, ${ }^{21}$ M. Bishai,${ }^{3}$ A. Blake, ${ }^{5}$ G. J. Bock, ${ }^{8}$ D. Bogert, ${ }^{8}$ S. V. Cao, ${ }^{29}$ D. Cherdack, ${ }^{30}$ S. Childress, ${ }^{8}$ J. A. B. Coelho, ${ }^{30,6}$ L. Corwin,${ }^{14}$ D. Cronin-Hennessy, ${ }^{18}$ J. K. de Jong, ${ }^{21}$ A. V. Devan, ${ }^{32}$ N.E. Devenish ${ }^{27}$ M. V. Diwan, ${ }^{3}$ C. O. Escobar, ${ }^{6}$ J. J. Evans,${ }^{17,16}$ E. Falk,${ }^{27}$ G. J. Feldman, ${ }^{10}$ M. V. Frohne, ${ }^{11}$ H. R. Gallagher, ${ }^{30}$ R. A. Gomes, ${ }^{9}$ M. C. Goodman, ${ }^{1}$ P. Gouffon, ${ }^{24}$ N. Graf, ${ }^{13}$ R. Gran, ${ }^{19}$ K. Grzelak,${ }^{31}$ A. Habig, ${ }^{19}$ J. Hartnell, ${ }^{27}$ R. Hatcher, ${ }^{8}$ A. Himmel,${ }^{4}$ A. Holin, ${ }^{16}$ J. Hylen, ${ }^{8}$ G. M. Irwin,${ }^{26}$ Z. Isvan, ${ }^{3,22}$ C. James, ${ }^{8}$ D. Jensen, ${ }^{8}$ T. Kafka, ${ }^{30}$ S. M. S. Kasahara, ${ }^{18}$ G. Koizumi, ${ }^{8}$ M. Kordosky, ${ }^{32}$ A. Kreymer ${ }^{8}$ K. Lang, ${ }^{29}$ J. Ling, ${ }^{3}$ P. J. Litchfield,${ }^{18,23}$ P. Lucas, ${ }^{8}$ W. A. Mann, ${ }^{30}$ M. L. Marshak, ${ }^{18}$ M. Mathis, ${ }^{32}$ N. Mayer,${ }^{30,14}$ M. M. Medeiros, ${ }^{9}$ R. Mehdiyev, ${ }^{29}$ J. R. Meier, ${ }^{18}$ M. D. Messier, ${ }^{14}$ W. H. Miller, ${ }^{18}$ S. R. Mishra, ${ }^{25}$ S. Moed Sher, ${ }^{8}$ C. D. Moore, ${ }^{8}$ L. Mualem,${ }^{4}$ S. Mufson, ${ }^{14}$ J. Musser, ${ }^{14}$ D. Naples, ${ }^{22}$ J. K. Nelson, ${ }^{32}$ H. B. Newman, ${ }^{4}$ R. J. Nichol, ${ }^{16}$ J. A. Nowak, ${ }^{18}$ W. P. Oliver, ${ }^{30}$ M. Orchanian, ${ }^{4}$ R. B. Pahlka, ${ }^{8}$ J. Paley, ${ }^{1}$ R. B. Patterson, ${ }^{4}$ G. Pawloski, ${ }^{18,26}$ S. Phan-Budd, ${ }^{1}$ R. K. Plunkett, ${ }^{8}$ X. Qiu, ${ }^{26}$ A. Radovic, ${ }^{16}$ B. Rebel,${ }^{8}$ C. Rosenfeld,${ }^{25}$ H. A. Rubin, ${ }^{13}$ M. C. Sanchez,${ }^{15,1}$ J. Schneps,${ }^{30}$ A. Schreckenberger ${ }^{18}$ P. Schreiner, ${ }^{1}$ R. Sharma, ${ }^{8}$ A. Sousa,${ }^{7,10}$ N. Tagg, ${ }^{20}$ R. L. Talaga, ${ }^{1}$ J. Thomas,${ }^{16}$ M. A. Thomson, ${ }^{5}$ R. Toner, ${ }^{10,5}$ D. Torretta, ${ }^{8}$ G. Tzanakos, ${ }^{2}$ J. Urheim, ${ }^{14}$ P. Vahle, ${ }^{32}$ B. Viren, ${ }^{3}$ A. Weber ${ }^{21,23}$ R. C. Webb, ${ }^{28}$ C. White, ${ }^{13}$ L. Whitehead, ${ }^{12,3}$ S. G. Wojcicki, ${ }^{26}$ and R. Zwaska ${ }^{8}$

\section{(MINOS Collaboration)}

\author{
${ }^{1}$ Argonne National Laboratory, Argonne, Illinois 60439, USA \\ ${ }^{2}$ Department of Physics, University of Athens, GR-15771 Athens, Greece \\ ${ }^{3}$ Brookhaven National Laboratory, Upton, New York 11973, USA \\ ${ }^{4}$ Lauritsen Laboratory, California Institute of Technology, Pasadena, California 91125, USA \\ ${ }^{5}$ Cavendish Laboratory, University of Cambridge, Madingley Road, Cambridge CB3 OHE, United Kingdom \\ ${ }^{6}$ Universidade Estadual de Campinas, IFGW-UNICAMP, CP 6165, 13083-970 Campinas, São Paulo, Brazil \\ ${ }^{7}$ Department of Physics, University of Cincinnati, Cincinnati, Ohio 45221, USA \\ ${ }^{8}$ Fermi National Accelerator Laboratory, Batavia, Illinois 60510, USA \\ ${ }^{9}$ Instituto de Física, Universidade Federal de Goiás, CP 131, 74001-970 Goiânia, Goiás, Brazil \\ ${ }^{10}$ Department of Physics, Harvard University, Cambridge, Massachusetts 02138, USA \\ ${ }^{11}$ Holy Cross College, Notre Dame, Indiana 46556, USA \\ ${ }^{12}$ Department of Physics, University of Houston, Houston, Texas 77204, USA \\ ${ }^{13}$ Department of Physics, Illinois Institute of Technology, Chicago, Illinois 60616, USA \\ ${ }^{14}$ Indiana University, Bloomington, Indiana 47405, USA \\ ${ }^{15}$ Department of Physics and Astronomy, Iowa State University, Ames, Iowa 50011 USA \\ ${ }^{16}$ Department of Physics and Astronomy, University College London, Gower Street, \\ London WC1E 6BT, United Kingdom \\ ${ }^{17}$ School of Physics and Astronomy, University of Manchester, Oxford Road, \\ Manchester M13 9PL, United Kingdom \\ ${ }^{18}$ University of Minnesota, Minneapolis, Minnesota 55455, USA \\ ${ }^{19}$ Department of Physics, University of Minnesota Duluth, Duluth, Minnesota 55812, USA \\ ${ }^{20}$ Otterbein College, Westerville, Ohio 43081, USA \\ ${ }^{21}$ Subdepartment of Particle Physics, University of Oxford, Oxford OX1 3RH, United Kingdom \\ ${ }^{22}$ Department of Physics and Astronomy, University of Pittsburgh, Pittsburgh, \\ Pennsylvania 15260, USA \\ ${ }^{23}$ Rutherford Appleton Laboratory, Science and Technologies Facilities Council, \\ OX11 0QX, United Kingdom \\ ${ }^{24}$ Instituto de Física, Universidade de São Paulo, CP 66318, 05315-970 São Paulo, SP, Brazil \\ ${ }^{25}$ Department of Physics and Astronomy, University of South Carolina, Columbia, \\ South Carolina 29208, USA \\ ${ }^{26}$ Department of Physics, Stanford University, Stanford, California 94305, USA \\ ${ }^{27}$ Department of Physics and Astronomy, University of Sussex, Falmer, Brighton BN1 9QH, United Kingdom \\ ${ }^{28}$ Physics Department, Texas A\&M University, College Station, Texas 77843, USA \\ ${ }^{29}$ Department of Physics, University of Texas at Austin, 1 University Station C1600, Austin, Texas 78712, USA \\ ${ }^{30}$ Physics Department, Tufts University, Medford, Massachusetts 02155, USA \\ ${ }^{31}$ Department of Physics, University of Warsaw, Hoża 69, PL-00-681 Warsaw, Poland \\ ${ }^{32}$ Department of Physics, College of William \& Mary, Williamsburg, Virginia 23187, USA
}

(Received 22 March 2013; published 31 October 2013) 
We report new constraints on flavor-changing non-standard neutrino interactions from the MINOS experiment, in which neutrino versus antineutrino interactions can be distinguished on an event-by-event basis. We analyzed a combined set of beam neutrino and antineutrino data from the well-understood NuMI beam, and found no evidence for deviations from standard neutrino mixing. The observed energy spectra constrain the non-standard neutrino interactions parameter to the range $-0.20<\varepsilon_{\mu \tau}<$ 0.07 (90\%C.L.).

DOI: 10.1103/PhysRevD.88.072011

It is well established from solar, atmospheric, reactor and accelerator experiments [1-7] that neutrinos undergo flavor change as they propagate. This phenomenon can be explained by the quantum mechanical mixing of neutrino flavor and mass eigenstates. The mixing can be parametrized by three angles, $\theta_{12}, \theta_{13}, \theta_{23}$, and a $C P$-violating phase, $\delta$ [8]. The standard neutrino oscillation mechanism requires that at least two of the three active neutrinos are massive. While the phenomenon of oscillations can occur in vacuum, the presence of matter along the neutrino path allows for alternative flavor changing mechanisms such as the Mikheyev-Smirnov-Wolfenstein (MSW) matter effect [9]. This effect alters the survival probability of electron neutrinos propagating through matter since electron neutrinos can have additional interactions with electrons in the surrounding medium, but does not affect the survival probability of muon or tau neutrinos.

Non-standard interactions (NSI) [10] could occur between muon or tau neutrinos and matter, and could alter the flavor content of a neutrino beam as it propagates through the Earth's crust in a manner similar to standard matter effects. Searches for NSI have already been performed with atmospheric neutrinos [11]. However, non-standard matter effects are, in general, different for neutrinos and antineutrinos. Accelerator-based oscillation experiments offer a powerful tool to search for NSI with their ability to produce well-understood beams of neutrinos and antineutrinos separately. Furthermore, MSW and NSI effects depend on the neutrino's path length in matter, and beam neutrinos travel a well-defined distance to the detector.

A NSI search using data from an atmospheric neutrino experiment requires a three-flavor formalism with multiple NSI due to the wide range of baselines and large value of $\theta_{13}$. MINOS, on the other hand, can isolate NSI that affect flavor change in the mu-tau sector; the electron-tau mixing sector is effectively disjoint from the mu-tau sector at the MINOS baseline [12,13]. Furthermore, the magnetized MINOS detectors have the capability of identifying neutrinos and antineutrinos on an event-by-event basis. This feature and the use of a well-controlled and well-modeled neutrino beam make MINOS well-suited to constrain NSI of the mu-tau mixing sector. Recent papers have discussed the compatibility of NSI with a smaller set of MINOS data $[14,15]$. This paper describes the first direct search for NSI which simultaneously fits the separate energy spectra of neutrinos and antineutrinos in a long-baseline experiment,
PACS numbers: 13.15.+g, 14.60.Lm, 14.60.Pq, 14.60.St

using a factor of two larger antineutrino sample than $[14,15]$.

Short-baseline neutrino experiments have explored and constrained non-standard interactions [16]. In such experiments both charged-current (CC) and neutral-current (NC) NSI can be studied. While long-baseline experiments can also constrain interactions with matter directly in either near or far detectors, their improved sensitivity arises from using the Earth's matter along the long neutrino path as the interaction medium. Thus, we focus only on NSI during propagation. Charged-current NSI that occur along the propagation path produce a final state charged-lepton which is absorbed by the Earth's matter and not observed. However, NC NSI that occur along the neutrino path will produce a final state neutrino of a different flavor, altering the flavor content of the beam $[15,17]$. We consider only NC NSI in this study.

The NSI Hamiltonian can be included as a perturbation to standard oscillations. This Hamiltonian is proportional to the matter potential $V=\sqrt{2} G_{F} N_{e}$, with $G_{F}$, the Fermi coupling constant, and $N_{e}$, the electron density in matter, analogous to the MSW matter effect [9]. In the two-flavor approximation the Hamiltonian is

$$
H_{\mathrm{NSI}}=V\left(\begin{array}{cc}
\varepsilon_{\mu \mu} & \varepsilon_{\mu \tau} \\
\varepsilon_{\mu \tau}^{*} & \varepsilon_{\tau \tau}
\end{array}\right)
$$

where the coefficients $\varepsilon_{\alpha \beta}$ give the strength of the NSI effect on transitions between $\alpha$ and $\beta$ flavors. In general, the Hamiltonian has both flavor-conserving and flavorchanging components, the diagonal and off-diagonal terms of the Hamiltonian, respectively. In flavor-conserving NSI, the NC scattering between the neutrino and matter does not alter the neutrino flavor. Flavor-changing NSI, on the other hand, do not conserve lepton flavor number; the final state neutrino is in a different flavor eigenstate from the initial neutrino. In a disappearance experiment, flavor-changing NSI have a greater effect on the flavor transition probability than flavor-conserving NSI due to interference between amplitudes of standard and non-standard matter interactions [18]. We verified this assertion by including both flavor-conserving and flavor-changing terms in our analysis, and found that the flavor-conserving components have a negligible impact on the result. We consider here only flavor-changing NSI and set to zero flavor-conserving amplitudes to which MINOS has no sensitivity.

We only consider the real part of $\varepsilon_{\mu \tau}$, which is sensitive to differences between $\nu_{\mu}$ and $\bar{\nu}_{\mu}$ survival. We define the 
vacuum oscillation length for neutrinos of energy $E$ as $L_{0} \equiv$ $\left(\frac{4 E}{\Delta m^{2}}\right)$. The difference between the squares of the second and third neutrino masses, $\Delta m^{2}=\Delta m_{32}^{2} \equiv m_{3}^{2}-m_{2}^{2}$, is the same parameter that governs standard neutrino oscillations $[19,20]$. The NSI matter oscillation length is defined as

$$
L_{m} \equiv \frac{L_{0}}{\left[1 \pm 2 \sin (2 \theta) L_{0} \varepsilon_{\mu \tau}|V|+\left(L_{0} \varepsilon_{\mu \tau}|V|\right)^{2}\right]^{\frac{1}{2}}} .
$$

The survival probability can then be written as

$$
P\left(\nu_{\mu} \rightarrow \nu_{\mu}\right)=1-\left[1-\cos ^{2}(2 \theta) \frac{L_{m}^{2}}{L_{0}^{2}}\right] \sin ^{2}\left(\frac{L}{L_{m}}\right),
$$

with mixing angle $\theta=\theta_{23}$, and neutrino path length $L$. Standard oscillation parameters $\theta$ and $\Delta m^{2}$ are taken to be the same for neutrinos and antineutrinos. The \pm signs in Eq. (2) arise from the matter potential, $V$, which is positive for neutrinos and negative for antineutrinos. The parameter $\varepsilon_{\mu \tau}$ is real-valued and carries its own sign. A positive value of $\varepsilon_{\mu \tau}$ implies that the neutrino disappearance probability is greater than the antineutrino disappearance probability, and vice versa.

The MINOS experiment measures the disappearance of muon neutrinos and antineutrinos in the NuMI beam [21] using two detectors. The event energy spectrum of the low energy NuMI beam, used in this measurement, peaks at approximately $3 \mathrm{GeV}$ [22]. Its focusing components can be tuned to produce a beam with an event composition of $91.7 \% \nu_{\mu}, 7 \% \bar{\nu}_{\mu}$, and $1.3 \%\left(\nu_{e}+\bar{\nu}_{e}\right)$ in neutrino mode, or of $58 \% \nu_{\mu}, 40 \% \bar{\nu}_{\mu}$, and $2 \%\left(\nu_{e}+\bar{\nu}_{e}\right)$ in antineutrino mode [23]. The Near Detector (ND), with a fiducial mass of 23.7 tons, measures the neutrino and antineutrino energy spectra $1.04 \mathrm{~km}$ downstream of the production target. The Far Detector (FD) is located in the Soudan Underground Laboratory and has a 4.2 kiloton fiducial mass. It measures the energy spectra $735 \mathrm{~km}$ downstream of the production target. Both detectors are magnetized steel-scintillator tracking-sampling calorimeters designed to measure the energy and the sign of the charge of muons produced by $\nu_{\mu}$ and $\bar{\nu}_{\mu}$ interactions. In each detector, muon neutrino and antineutrino CC interactions are separated event-byevent using the sign of muon track curvature.

The results presented here are based on an exposure of $7.09 \times 10^{20}$ protons on target (POT) in neutrino mode, combined with a $2.95 \times 10^{20}$ POT exposure in antineutrino mode, doubling the antineutrino data set of $[14,15]$. MINOS previously reported a two-flavor oscillation analysis on this neutrino data set $[19,20]$ and the first direct measurement of antineutrino oscillation parameters from the antineutrino sample [23]. Due to the opposite sign of the matter potential in Eq. (3) for neutrinos and antineutrinos, NSI, if present, will alter the survival probability of neutrinos and antineutrinos in opposite directions. The magnitude of $\varepsilon_{\mu \tau}$ is proportional to the difference in probability between neutrinos and antineutrinos, and the sign of $\varepsilon_{\mu \tau}$ is determined by the sign of the probability difference.

We select $\nu_{\mu}$ and $\bar{\nu}_{\mu} \mathrm{CC}$ events inside the fiducial volume by identifying interaction vertices with a muon track and possible hadronic activity. The neutrino energy is reconstructed by summing the muon track and hadronic shower energies. Muon energy is measured using range for muons that stop in the detector and curvature for muons that exit. The hadronic energy is determined using a $k$-nearest neighbor $(k N N)$ technique [24]. We require the muon charge be negative for $\nu_{\mu}$ and positive for $\bar{\nu}_{\mu}$ events.

To reject NC interactions we use a discriminant obtained by combining four event characteristics into a $k \mathrm{NN}$ variable [25]. The selection criteria optimize selection efficiency and sample purity to obtain maximum sensitivity to oscillations [24,26]. Far Detector selection efficiencies for neutrino and antineutrino samples are 93\% and 97\%, with purities of $99 \%$ and $94 \%$, respectively. Because the neutrino sample has a larger component of highly inelastic events, this optimization process reduces the optimal neutrino selection efficiency in favor of lower NC background. The lower overall purity of the antineutrino sample results from the much larger neutrino contamination at higher energies in the antineutrino mode (wrong-sign background); however, in the region of interest to oscillations and NSI the contamination is smaller.

The FD neutrino and antineutrino spectra in the absence of flavor change are predicted using the ND data by first correcting the ND spectra for inefficiency and backgrounds and then extrapolating to the FD by a transfer matrix obtained from simulation $[3,27]$. We predict 2073 neutrino and 273 antineutrino events without oscillations, and observe 1654 and 193 events, respectively.

The neutrino and antineutrino energy spectra are fit simultaneously to three parameters, $\left|\Delta m^{2}\right|, \sin ^{2} 2 \theta$, and $\varepsilon_{\mu \tau}$, in the combined oscillation and NSI model in Eq. (3), using a binned log-likelihood. The value of the mixing angle is constrained to be physical by asserting $0 \leq \sin ^{2}(2 \theta) \leq 1$. The resulting simulated energy spectra, obtained by using the best fit values, are shown in Fig. 1 superimposed on the full neutrino and antineutrino spectra.

The overall systematic uncertainty in the measurement is much smaller than the statistical uncertainty. However, the difference in the relative sizes of the neutrino and antineutrino event samples results in a large difference in their statistical uncertainties, while the systematic uncertainties are comparable. Systematic uncertainties are included in the fit to ensure that the neutrino sample does not have a disproportionate impact.

There are four significant sources of systematic uncertainty: (i) the hadronic energy scale, (ii) the muon energy scale, (iii) the NC background, and (iv) the relative normalization between the Near and Far detectors. Correlations in systematic effects between neutrino and antineutrino data samples are taken into account in the evaluation of 
uncertainties. Uncertainties in wrong-sign background, cross section model, and flux extrapolation were also considered, but were found to be insignificant and have been neglected.

The hadronic energy scale uncertainty has multiple components which arise from modeling of underlying distributions and spectra of hadrons produced in neutrino interactions and from knowledge of the detector response to interacting hadrons. The hadronic detector response was measured in a test beam [28] and found to have a 5.7\% uncertainty that is constant with energy, (including a small component that accounts for detector calibration uncertainty obtained using cosmic muons). Uncertainty in the hadronization and shower modeling contributions peak at low energies and fall off exponentially. The overall uncertainty, parametrized by

$$
\sigma_{\text {had }}=6.6 \%+3.5 \% \exp \left(\frac{-E_{\text {had }}}{1.44 \mathrm{GeV}}\right) \text {, }
$$
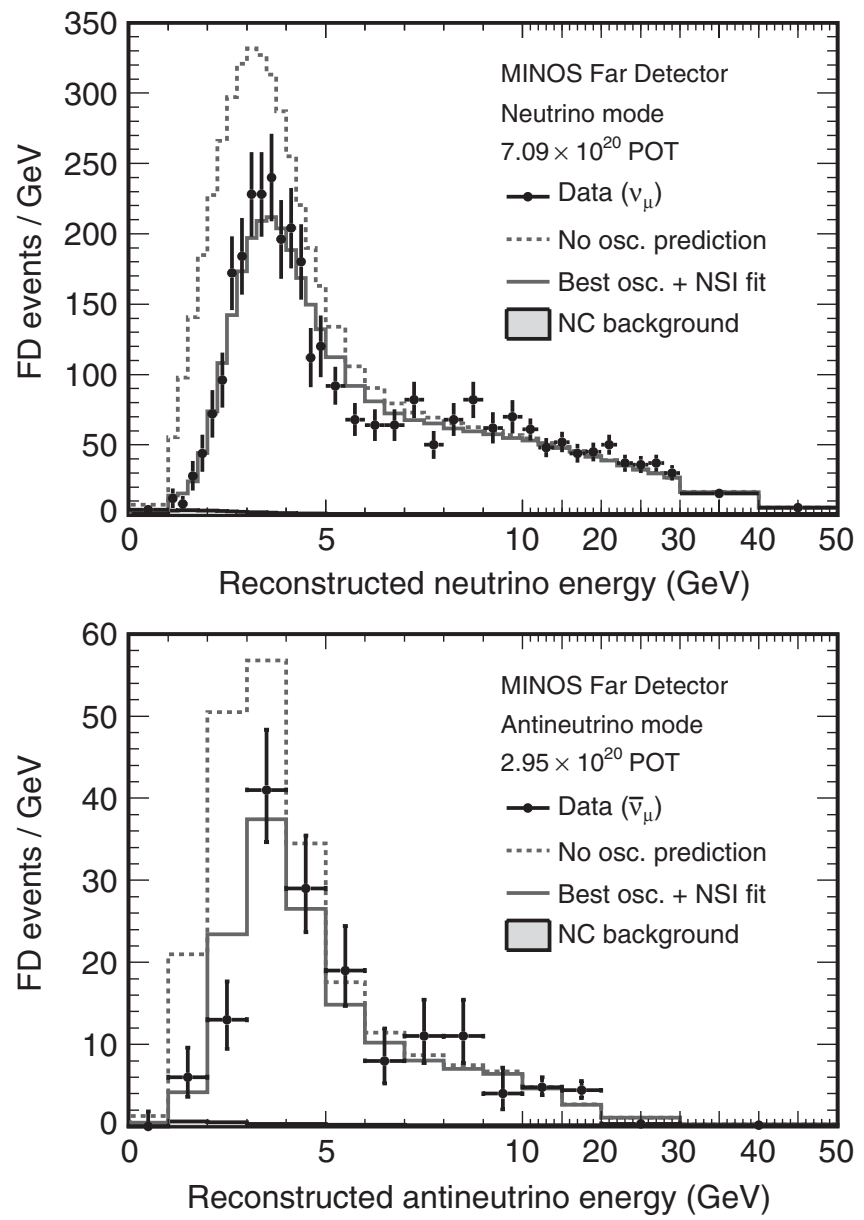

FIG. 1. Far Detector distributions of selected neutrino (top) and antineutrino (bottom) events. Black dots represent data, the dashed histogram shows the prediction in the absence of oscillations and the solid histogram shows the prediction for the model in Eq. (3) at the values obtained in our fit to the oscillation and NSI model. is obtained by combining the effects in quadrature. Uncertainty in the modeling contribution will be reduced in the future with precise final-state measurements from fine-grained neutrino detectors.

The muon energy scale uncertainty is $2 \%$ for events where muon energy is determined from track range and $3 \%$ where the energy is obtained by measuring the track curvature in the magnetic field. The uncertainty from range is based on a comparison of tabulated muon spectrum
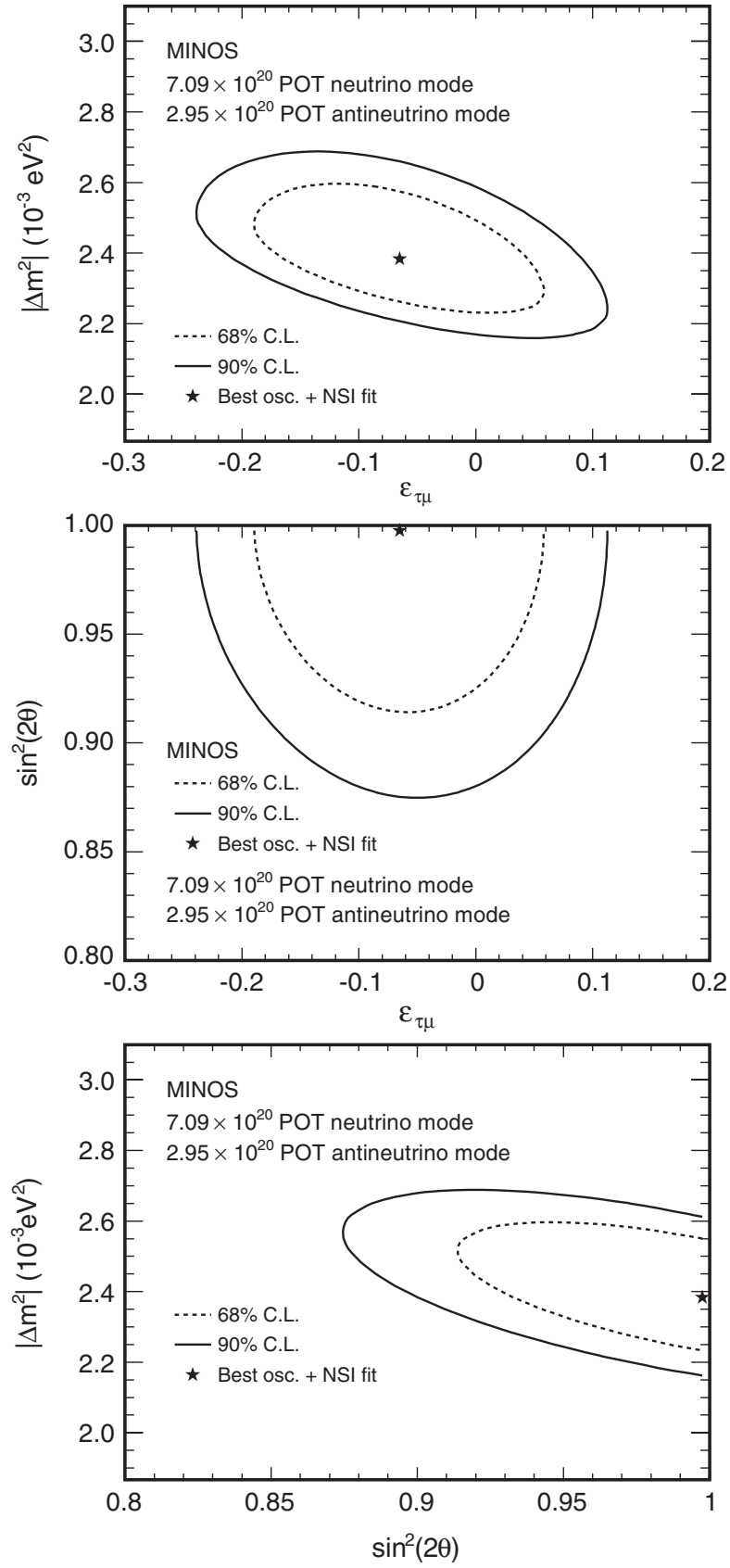

FIG. 2. The $68 \%$ and $90 \%$ allowed regions for the three parameters in the combined oscillation and NSI flavor change model given in Eq. (3). To obtain slices for each parameter pair combination we marginalize over the third parameter as well as over the systematic penalty parameters. 
power in the MINOS detectors with tables in [29], and includes uncertainty in the material composition of the detectors. The uncertainty in momentum from curvature is found by comparing the curvature measurement to that from range for tracks that end in the detector [19].

The NC background is less than $2 \%$ of both the neutrino and antineutrino samples integrated across all energies. The size of its uncertainty, which is dominated by hadronic shower modeling, is estimated to be $20 \%$ by comparing a muon-removed CC event sample in Monte Carlo simulation, and scaling it to match the data [30].

There are differences in normalization and modeling of event samples recorded by the Near versus Far detectors to which a relative normalization uncertainty of $1.6 \%$ is assigned [19]. The largest component (1.3\%) is estimated by tabulating results of a manual event scan of a large sample of events to quantify our modeling of reconstruction differences between Near and Far detectors. Other smaller contributions are also accounted for and include Far Detector live time, detector material accounting, and Near Detector fiducial geometry modeling.

These four sources of systematic uncertainty are included in the fit using penalty terms. The best fit parameters from this procedure are found to be

$$
\begin{gathered}
\Delta m^{2}=2.39_{-0.11}^{+0.14} \times 10^{-3} \mathrm{eV}^{2}, \quad \sin ^{2}(2 \theta)=1.00_{-0.06}^{+0.00}, \\
\varepsilon_{\mu \tau}=-0.07_{-0.08}^{+0.08}
\end{gathered}
$$

with the allowed region $-0.20<\varepsilon_{\mu \tau}<0.07$ (90\% C.L.).
The penalty terms from systematic uncertainties have a negligible effect on the fit; each penalty term pulls the best fit point by much less than one standard deviation. The allowed regions of fit parameters are shown in Fig. 2, where three two-dimensional slices from a 3D likelihood surface are chosen by marginalizing over the third parameter. The obtained oscillation parameter values are in good agreement with previously published results in $[19,23]$. Within errors the fit is consistent with no contribution to flavor change from NSI. This result is in agreement with recent results from the Super-Kamiokande collaboration who report $\left|\varepsilon_{\mu \tau}\right|<1.1 \times 10^{-2}$ at $90 \%$ C.L. [11], as well as with values of $\varepsilon_{\mu \tau}$ extracted from global fits to data from multiple experiments [31-34].

In summary, this is the first direct search for nonstandard interactions with high-purity samples of both neutrinos and antineutrinos. We conducted a simultaneous fit to neutrino and antineutrino energy spectra of conventional $\nu_{\mu} \rightarrow \nu_{\tau}$ oscillations with an additional NSI matter effect. We found no evidence for non-standard neutrino interactions.

This work was supported by the U.S. DOE; the United Kingdom STFC; the U.S. NSF; the State and University of Minnesota; the University of Athens, Greece; and Brazil's FAPESP, CNPq and CAPES. We are grateful to the Minnesota Department of Natural Resources, the crew of the Soudan Underground Lab, and the personnel of Fermilab for their contribution to this effort.
[1] Y. Ashie et al. (Super-Kamiokande), Phys. Rev. Lett. 93, 101801 (2004); Phys. Rev. D 71, 112005 (2005).

[2] B. Aharmin et al. (SNO), Phys. Rev. C 72, 055502 (2005).

[3] D. G. Michael et al. (MINOS), Phys. Rev. Lett. 97, 191801 (2006); P. Adamson et al. (MINOS), Phys. Rev. D 77, 072002 (2008).

[4] T. Araki et al. (KamLAND), Phys. Rev. Lett. 94, 081801 (2005).

[5] C. Arpesella et al. (Borexino), Phys. Rev. Lett. 101, 091302 (2008).

[6] M. H. Ahn et al. (K2K), Phys. Rev. D 74, 072003 (2006).

[7] F. P. An et al. (Daya Bay), Phys. Rev. Lett. 108, 171803 (2012); J. K. Ahn et al. (RENO), Phys. Rev. Lett. 108, 191802 (2012).

[8] B. Pontecorvo, JETP 34, 172 (1958); V. N. Gribov and B. Pontecorvo, Phys. Lett. 28B, 493 (1969); Z. Maki, M. Nakagawa, and S. Sakata, Prog. Theor. Phys. 28, 870 (1962).

[9] L. Wolfenstein, Phys. Rev. D 17, 2369 (1978); S. P. Mikheyev and A. Yu. Smirnov, Sov. J. Nucl. Phys. 42, 913 (1985).

[10] L. Wolfenstein, Phys. Rev. D 17, 2369 (1978); J. W. F. Valle, Phys. Lett. B 199, 432 (1987); M. Gonzalez-Garcia,
M. Guzzo, P. Krastev, H. Nunokawa, O. Peres, V. Pleitez, J. Valle, and R. Zukanovich Funchal, Phys. Rev. Lett. 82, 3202 (1999); A. Friedland, C. Lunardini, and M. Maltoni, Phys. Rev. D 70, 111301 (2004).

[11] G. Mitsuka et al. (Super-Kamiokande), Phys. Rev. D 84, 113008 (2011).

[12] H. Minakata, in Proceedings of the 13th Int. Workshop on Neutrino Telescopes, edited by Milla Baldo Ceolin Venice, Italy, March 2009, p. 327.

[13] K. Asano and H. Minakata, J. High Energy Phys. 06 (2011) 022.

[14] W. A. Mann, D. Cherdack, W. Musial, and T. Kafka, Phys. Rev. D 82, 113010 (2010).

[15] J. Kopp, P. A. N. Machado, and S. J. Parke, Phys. Rev. D 82, 113002 (2010).

[16] G. P. Zeller et al. (NuTeV), Phys. Rev. Lett. 88, 091802 (2002); S. Davidson, S. Forte, P. Gambino, N. Rius, and A. Strumia, J. High Energy Phys. 02 (2002) 037.

[17] T. Ohlsson, Rep. Prog. Phys. 76, 044201 (2013).

[18] J. Kopp, M. Lindner, T. Ota, and J. Sato, Phys. Rev. D 77, 013007 (2008).

[19] P. Adamson et al. (MINOS), Phys. Rev. Lett. 106, 181801 (2011). 
[20] The experiment measures an unresolved mixture of $\left|\Delta m_{31}^{2}\right|$ and $\left|\Delta m_{32}^{2}\right|$, which is referred to as $\left|\Delta m^{2}\right|$ for brevity. The parameter $\sin ^{2}(2 \theta)$ is likewise an admixture, dominated by $\theta_{23}$.

[21] K. Anderson et al., Report No. FERMILAB-DESIGN1998-01, 1998.

[22] D. G. Michael et al., Nucl. Instrum. Methods Phys. Res., Sect. A 596, 190 (2008).

[23] P. Adamson et al. (MINOS), Phys. Rev. Lett. 107, 021801 (2011); P. Adamson et al. (MINOS), Phys. Rev. Lett. 108, 191801 (2012).

[24] C. Backhouse, D.Phil. thesis, University of Oxford, 2011.

[25] R. Ospanov, Ph.D. thesis, University of Texas at Austin, 2008.

[26] Z. Isvan, Ph.D. thesis, University of Pittsburgh, 2012.
[27] J. J. Evans, D.Phil. thesis, University of Oxford, 2008.

[28] M. A. Kordosky, Ph.D. thesis, University of Texas, Austin, 2004.

[29] D. E. Groom, N. V. Mokhov, and S. I. Striganov, Atom. Data Nucl. Data Tabl. 78, 183 (2001).

[30] J. Ratchford, Ph.D. thesis, University of Texas, Austin, 2011.

[31] M. Blennow, T. Ohlsson, and J. Skrotzki, Phys. Lett. B 660, 522 (2008).

[32] M. Blennow, T. Ohlsson, and W. Winter, Eur. Phys. J. C 49, 1023 (2007).

[33] C. Biggio, M. Blennow, and E. Fernández-Martínez, J. High Energy Phys. 08 (2009) 090.

[34] F. J. Escrihuela, M. Tortola, J. W. F. Valle, and O. G. Miranda, Phys. Rev. D 83, 093002 (2011). 